\title{
Continuous solutions of 3rd-order iterative equation of linear dependence
}

\author{
Pingping Zhang ${ }^{1 *}$ and Xiaobing Gong ${ }^{2 *}$
}

"Correspondence:

zhangpingpingmath@163.com;

xbgong@163.com

'Department of Mathematics,

Binzhou University, Binzhou,

Shandong 256603, China

2Department of Mathematics,

Neijiang Normal University,

Neijiang, Sichuan 641100, China

\begin{abstract}
We study continuous solutions of the 3rd-order iterative equation of the linear dependence for hyperbolic cases and generalize the results to the $n$ th-order polynomial-like iterative equation, in some cases recursively. This paper partly answers the question raised in Remark 7 in (Yang and Zhang in Aequ. Math. 67:80-105, 2004) and the open problem raised by Matkowski in (Aequ. Math. 37:119, $1989)$ in the 26th international symposium on functional equations.
\end{abstract}

MSC: 39B12; 37E05

Keywords: functional equation; iteration; characteristic root; continuous solution

\section{Introduction}

Consider a real function $f: \mathbb{R} \rightarrow \mathbb{R}$. Its $n$th iterate is defined by $f^{n}(x)=f\left(f^{n-1}(x)\right), f^{0}(x)=$ $x$ for all $x \in \mathbb{R}$ recursively. A functional equation involving the iterates of the unknown function is called an iterative functional equation. Some problems of dynamical systems such as iterative root $[1,2]$ and invariant curve $[3,4]$ reduce to those iterative equations, a more general and important form is the polynomial-like iterative equation

$$
\lambda_{n} f^{n}(x)+\lambda_{n-1} f^{n-1}(x)+\cdots+\lambda_{1} f(x)=F(x)
$$

an equation of the linear dependence of iterates. While attention was paid to the case of nonlinear $F$ (see e.g. [5-11]) and further generalized forms [12,13], the case of linear $F$, that is,

$$
f^{n}(x)+\lambda_{n-1} f^{n-1}(x)+\cdots+\lambda_{1} f(x)+\lambda_{0} x=c,
$$

even the homogeneous equation

$$
f^{n}(x)+\lambda_{n-1} f^{n-1}(x)+\cdots+\lambda_{1} f(x)+\lambda_{0} x=0
$$

is also fascinating. In 1974 Nabeya [14] investigated the generalized Euler equation $f(p+$ $q x+r f(x))=a+b x+c f(x)$, which actually is equivalent to (1.2) for $n=2$. He showed all iterates $f^{k}$ by a system of linear homogeneous difference equations and formulated continuous solutions by this system's eigenvalues. However, all continuous solutions of (1.2) for $n \geq 3$ have long been in suspense. In 1989 Matkowski [15] raised an open problem

\section{Springer}

@2014 Zhang and Gong; licensee Springer. This is an Open Access article distributed under the terms of the Creative Commons Attribution License (http://creativecommons.org/licenses/by/2.0), which permits unrestricted use, distribution, and reproduction in any medium, provided the original work is properly cited. 
in the 26th international symposium on functional equations: 'How to determine all the continuous solutions $f: \mathbb{R} \rightarrow \mathbb{R}$ of the nth-order homogeneous iterative equation under the condition $\lambda_{0} \neq 0$, even for $n=3$ ?', which has attracted attention of many scholars.

Let $r_{1}, r_{2}, \ldots, r_{n}$ be $n$ complex roots of the characteristic equation

$$
P(r):=r^{n}+\lambda_{n-1} r^{n-1}+\cdots+\lambda_{1} r+\lambda_{0}=0 .
$$

In 1996 Jarczyk [16] discussed (1.3) defined on the interval not containing zero and gave the linear solution $f=c x$, where $\lambda_{i}>0>\lambda_{0}(i=1, \ldots, n-1)$ and $c$ is the unique positive characteristic root. Tabor and Tabor [17] investigated (1.3) defined on the same domain. Assuming that $r$ is the unique positive and simple characteristic root and is smaller than the module of all complex ones, they proved that the equation has exactly a solution $f(x)=r x$ depending continuously on all coefficients. In 1997 Matkowski and Zhang [18] established a characteristic theory of the second order (1.3) with $\lambda_{0} \neq 0$, i.e., all continuous solutions were represented by using two characteristic solutions $f(x)=r_{i} x(i=1,2)$.

Characteristic theory of (1.3) for $n \geq 3$, as we know, is more complicated. In 2004 Yang and Zhang [19] constructed all continuous solutions of (1.3) for the hyperbolic cases: (1) $1<r_{1}<r_{2}<\cdots<r_{n}$; (2) $0<r_{1}<r_{2}<\cdots<r_{n}<1$; (3) $r_{1}<r_{2}<\cdots<r_{n}<-1$; (4) $-1<r_{1}<r_{2}<\cdots<r_{n}<0$, and they proved no continuous solutions when the characteristic roots are all complex roots, and they gave a lowing order method for the $n$-multiple characteristic roots case. The authors pointed out [19, Remark 7] that when (1.3) has both positive characteristic roots and negative ones or some characteristic roots are greater than 1 in absolute value and the others less than 1 in absolute value, the construction of continuous solutions is not yet known. Recently, Zhang and Zhang [20, Lemma 3] indicated that a solution of (1.2) is a translation of that of (1.3) if all $r_{i} \neq 0,1(i=1,2, \ldots, n)$. The authors gave a method to lower the order of (1.3) when the module of all complex characteristic roots is larger than that of the real characteristic roots. Furthermore, the paper obtained the continuous solutions of (1.3) for the nonhyperbolic case $1=r_{1}<r_{2}<\cdots<r_{n}$, and proved no continuous solutions for (1.2) with $c \neq 0$ in the case of all characteristic roots being 1 .

So far, for $n \geq 3$ there are no results on the theory of characteristics for (1.3) in the following cases:

$\left(E_{+} C_{+}\right)$All characteristics are positive but some are greater than 1 and others are less than 1.

$\left(E_{-} C_{-}\right)$All characteristics are negative but some are greater than 1 in absolute value and others are less than 1 in absolute value.

$\left(E_{+} C_{-}\right)$Some characteristics are positive and greater than 1 but others are negative and less than 1 in absolute value.

$\left(E_{-} C_{+}\right)$Some characteristics are negative and greater than 1 in absolute value but others are positive and less than 1 .

$\left(C_{+} C_{-}\right)$Some characteristics are positive and less than 1 but others are negative and less than 1 in absolute value.

$\left(E_{+} E_{-}\right)$Some characteristics are positive and greater than 1 but others are negative and greater than 1 in absolute value.

Besides, there are cases including complex roots and many critical cases (a characteristic is equal to \pm 1 ) and degenerate cases (a characteristic vanishes). 
Following directly the idea from [19] and referring to [21, 22], in this paper we first get the general iterates $f^{m}$ of (1.3) with simple characteristic roots, where $m$ is a positive integer. Using this formula we construct all $C^{0}$ solutions of the homogeneous equation

$$
f^{3}(x)-\left(r_{1}+r_{2}+r_{3}\right) f^{2}(x)+\left(r_{1} r_{2}+r_{1} r_{3}+r_{2} r_{3}\right) f(x)-r_{1} r_{2} r_{3} x=0,
$$

where $\left|r_{i}\right| \neq 0,1(i=1,2,3)$ and have different modules. Those results are generalized to the $n$ th-order polynomial-like iterative equation, in some cases recursively. This paper is organized as follows: Section 2 introduces the properties of linear difference form and prove the generally iterative formula. Section 3 gives all continuous solutions for the cases $\left(E_{+} C_{+}\right),\left(E_{-} C_{-}\right)$, and Section 4 gives that of the cases $\left(E_{+} C_{-}\right),\left(E_{-} C_{+}\right),\left(C_{+} C_{-}\right),\left(E_{+} E_{-}\right)$. Finally, we give continuous solutions of the $n$ th-order polynomial-like iterative equation, in some cases. For convenience of statement, we write the dual equation of (1.4)

$$
f^{-3}(x)-\left(\frac{1}{r_{1}}+\frac{1}{r_{2}}+\frac{1}{r_{3}}\right) f^{-2}(x)+\left(\frac{1}{r_{1} r_{2}}+\frac{1}{r_{1} r_{3}}+\frac{1}{r_{2} r_{3}}\right) f^{-1}(x)-\frac{1}{r_{1} r_{2} r_{3}} x=0 .
$$

\section{Preliminaries}

In this section we state some notations and lemmas used in the proof of our results. Applying the linear difference form

$$
\mathcal{F}_{k}\left[r_{1}, \ldots, r_{n}\right]\left(x_{\ell}\right)_{\ell \in \mathbb{Z}}=\sum_{j=0}^{n}(-1)^{j} \sum_{1 \leq s_{1}<\cdots<s_{j} \leq n} r_{s_{1}} r_{s_{2}} \cdots r_{s_{j}} x_{k+n-j}
$$

first introduced in [23], where $\left(x_{\ell}\right)_{\ell \in \mathbb{Z}}:=\left(\ldots, x_{-1}, x_{0}, x_{1}, \ldots\right)$ is a bilateral sequence in vector space $X$ over $\mathbb{C},(1.2)$ is simplified to

$$
\mathcal{F}_{0}\left[r_{1}, \ldots, r_{n}\right]\left(f^{\ell}(x)\right)_{\ell \in \mathbb{Z}}=c, \quad \forall x \in \mathbb{R}
$$

Lemma 2.1 ([20, Lemma 1]) Suppose that $f: \mathbb{R} \rightarrow \mathbb{R}$ is a $C^{0}$ solution of $(2.1)$. If $\lambda_{0} \neq 0$, then $f$ is a homeomorphism.

Using Lemma 2.1, if $\lambda_{0} \neq 0$, the inverse $f^{-1}$ satisfies the dual equation

$$
\mathcal{F}_{0}\left[r_{1}^{-1}, \ldots, r_{n}^{-1}\right]\left(f^{-\ell}(x)\right)_{\ell \in \mathbb{Z}}=(-1)^{n} r_{1}^{-1} \cdots r_{n}^{-1} c, \quad \forall x \in \mathbb{R}
$$

Lemma 2.2 ([19, Lemmas 1, 2, 3], [20, Lemma 2]) Suppose that $n \geq 1$ and $k$ are integers, and $r_{1}, r_{2}, \ldots, r_{n}$ are complex numbers. If $\left(\tilde{r}_{1}, \ldots, \tilde{r}_{n}\right)$ is a permutation of $\left(r_{1}, \ldots, r_{n}\right)$, then $\mathcal{F}_{k}\left[\tilde{r}_{1}, \ldots, \tilde{r}_{n}\right]=\mathcal{F}_{k}\left[r_{1}, \ldots, r_{n}\right]$. Moreover,

(i) (Lower order) for integer $1 \leq q \leq n$,

$$
\mathcal{F}_{k}\left[r_{1}, \ldots, r_{n}\right]=\sum_{j=0}^{q}(-1)^{j} \sum_{n-q+1 \leq s_{1}<\cdots<s_{j} \leq n} r_{s_{1}} r_{s_{2}} \cdots r_{s_{j}} \mathcal{F}_{k+q-j}\left[r_{1}, \ldots, r_{n-q}\right]
$$

(ii) (Reduce to dual) if $r_{i} \neq 0(i=1,2, \ldots, n)$ then

$$
\mathcal{F}_{k}\left[r_{1}, \ldots, r_{n}\right]\left(f^{\ell}\right)_{\ell \in \mathbb{Z}}=(-1)^{n} r_{1} \cdots r_{n} \mathcal{F}_{-(k+n)}\left[r_{1}^{-1}, \ldots, r_{n}^{-1}\right]\left(f^{-\ell}\right)_{\ell \in \mathbb{Z}} ;
$$


(iii) (Shift on iteration) if the $C^{0}$ function $f: \mathbb{R} \rightarrow \mathbb{R}$ satisfies $\mathcal{F}_{0}\left[r_{1}, r_{2}, \ldots, r_{n}\right]\left(f^{\ell}\right)_{\ell \in \mathbb{Z}}=0$, then

$$
\mathcal{F}_{k+p}\left[r_{1}, \ldots, r_{t-1}, r_{t+1}, \ldots, r_{n}\right]\left(f^{\ell}\right)_{\ell \in \mathbb{Z}}=r_{t}^{p} \mathcal{F}_{k}\left[r_{1}, \ldots, r_{t-1}, r_{t+1}, \ldots, r_{n}\right]\left(f^{\ell}\right)_{\ell \in \mathbb{Z}}
$$

for arbitrary positive integers $p, t$ with $1 \leq t \leq n$.

Lemma 2.3 ([20, Lemma 3]) Suppose that all numbers $r_{i} \neq 0(i=1,2, \ldots, n)$ are real and none of them is equal to 1 . Then the equation $\mathcal{F}_{0}\left[r_{1}, r_{2}, \ldots, r_{n}\right]\left(f^{\ell}(x)\right)_{\ell \in \mathbb{Z}}=c$ for all $x \in|\alpha, \beta|$ can be reduced to the equation $\mathcal{F}_{0}\left[r_{1}, r_{2}, \ldots, r_{n}\right]\left(\tilde{f}^{\ell}(x)\right)_{\ell \in \mathbb{Z}}=0$ for all $x \in|\alpha-\xi, \beta-\xi|$ by the substitution $\tilde{f}(x)=f(x+\xi)-\xi$, where $\xi:=c / \prod_{\zeta=1}^{n}\left(1-r_{\varsigma}\right)$, and vice versa.

Lemma 2.4 If a $C^{0}$ solution $f: \mathbb{R} \rightarrow \mathbb{R}$ of (2.1) with $c=0$ has a nonzero fixed point, then at least one of its characteristic roots equals 1 .

Proof Assume that $f(\mu)=\mu \neq 0$. Substituting $x=\mu$ into (2.1) with $c=0$, we have $\mathcal{F}_{0}\left[r_{1}, \ldots, r_{n}\right](\mu)_{\ell \in \mathbb{Z}}=0$, i.e.,

$$
\prod_{i=1}^{n}\left(1-r_{i}\right) \mu=0
$$

Then one of $r_{i}(i=1,2, \ldots, n)$ equals 1 . This completes the proof.

Lemma 2.5 Suppose that the characteristic polynomial $P(r)$ has $n$ distinct roots $r_{1}, \ldots, r_{n}$ in $\mathbb{C}$. If $: \mathbb{R} \rightarrow \mathbb{R}$ is a $C^{0}$ solution of (2.1) with $c=0$, then

$$
\begin{aligned}
& f^{m}=\sum_{i=1}^{n} \frac{r_{i}^{m}}{\triangle\left(r_{1}, \ldots ; r_{i} ; \ldots, r_{n}\right)} \mathcal{F}_{0}\left[r_{1}, \ldots, r_{i-1}, r_{i+1}, \ldots, r_{n}\right]\left(f^{\ell}\right)_{\ell \in \mathbb{Z}} \\
& f^{-m}=\sum_{i=1}^{n} \frac{r_{i}^{-m}}{\triangle\left(r_{1}^{-1}, \ldots ; r_{i}^{-1} ; \ldots, r_{n}^{-1}\right)} \mathcal{F}_{0}\left[r_{1}^{-1}, \ldots, r_{i-1}^{-1}, r_{i+1}^{-1}, \ldots, r_{n}^{-1}\right]\left(f^{-\ell}\right)_{\ell \in \mathbb{Z}}
\end{aligned}
$$

for all integers $m \geq 1$ and $i=1,2, \ldots, n$, where $\triangle\left(r_{1}, \ldots ; r_{i} ; \ldots, r_{n}\right)=\prod_{j=1, j \neq i}^{n}\left(r_{i}-r_{j}\right)$.

Proof Let $f: \mathbb{R} \rightarrow \mathbb{R}$ be a $C^{0}$ solution of (2.1) with $c=0$, we have the shift on the iteration

$$
\mathcal{F}_{m}\left[r_{1}, \ldots, r_{i-1}, r_{i+1}, \ldots, r_{n}\right]\left(f^{\ell}\right)_{\ell \in \mathbb{Z}}=r_{i}^{m} \mathcal{F}_{0}\left[r_{1}, \ldots, r_{i-1}, r_{i+1}, \ldots, r_{n}\right]\left(f^{\ell}\right)_{\ell \in \mathbb{Z}}
$$

by using (iii) of Lemma 2.2. For fixed integer $m \geq 1$ and $i=1,2, \ldots, n,(2.5)$ is a system of $n$ linear equations for $f^{m+n-1}, f^{m+n-2}, \ldots, f^{m}$. Let

$$
\begin{aligned}
F & :=\left(f^{m+n-1}, f^{m+n-2}, \ldots, f^{m}\right)^{\tau}, \\
T & :=\left(r_{1}^{m} \mathcal{F}_{0}\left[r_{2}, \ldots, r_{n}\right]\left(f^{\ell}\right)_{\ell \in \mathbb{Z}}, \ldots, r_{n}^{m} \mathcal{F}_{0}\left[r_{1}, \ldots, r_{n-1}\right]\left(f^{\ell}\right)_{\ell \in \mathbb{Z}}\right),
\end{aligned}
$$

where ' $\tau$ ' stands for transposing a vector. Then (2.5) is rewritten as

$$
A F=T,
$$


in which

$$
A:=\left(\begin{array}{ccccc}
1 & -\sum_{j=2}^{n} r_{j} & \sum_{2 \leq j<k \leq n} r_{j} r_{k} & \cdots & (-1)^{n-1} r_{2} \cdots r_{n} \\
\vdots & \vdots & \vdots & \vdots & \vdots \\
\vdots & \vdots & \vdots & \vdots & \vdots \\
1 & -\sum_{j=1}^{n-1} r_{j} & \sum_{1 \leq j<k \leq n-1} r_{j} r_{k} & \cdots & (-1)^{n-1} r_{1} \cdots r_{n-1}
\end{array}\right) .
$$

In order to obtain $f^{m}$, our strategy is to transform $A$ into upper triangular matrix and every element of the principal diagonal is 1 . More precisely, multiplying the first row by -1 and then adding other rows, we see that the first element of the $i$ th $(2 \leq i \leq n)$ row equals 0 . Then for the $i$ th $(2 \leq i \leq n)$ row, we divide the second element and the matrix $A$ changes into

$$
\left(\begin{array}{ccccc}
1 & -\sum_{j=2}^{n} r_{j} & \sum_{2 \leq j<k \leq n} r_{j} r_{k} & \cdots & (-1)^{n-1} r_{2} \cdots r_{n} \\
0 & 1 & * & \cdots & * \\
\vdots & \vdots & \vdots & \vdots & \vdots \\
\vdots & \vdots & \vdots & \vdots & \vdots \\
0 & 1 & * & \cdots & *
\end{array}\right)
$$

where all $*$ are nonzero elements since $r_{i} \neq r_{j}$ for $1 \leq i, j \leq n$. Note that the submatrix

$$
\left(\begin{array}{cccc}
1 & * & \cdots & * \\
\vdots & \vdots & \vdots & \vdots \\
1 & * & \cdots & *
\end{array}\right)
$$

has the same form as that of $A$, using the same idea and arguments we obtain at last an upper triangular matrix such that

$$
\left(\begin{array}{ccccc}
1 & -\sum_{j=2}^{n} r_{j} & \sum_{2 \leq j<k \leq n} r_{j} r_{k} & \cdots & (-1)^{n-1} r_{2} \cdots r_{n} \\
0 & 1 & * & * & * \\
0 & 0 & 1 & * & * \\
\vdots & \vdots & \vdots & \vdots & \vdots \\
0 & 0 & 0 & \cdots & 1
\end{array}\right) .
$$

By using the above elementary invertible linear transformations, the augmented matrix $[A \mid T]$ transforms into row-echelon form, which yields (2.3) from the last low.

The proof of (2.4) is the same as that of (2.3) by considering the dual equation (2.2) and is omitted. This completes the proof.

From Lemma 2.5 we obtain the main tool used in our paper immediately.

Lemma 2.6 Suppose that (1.4) has three different characteristic roots $r_{1}, r_{2}, r_{3}$ in $\mathbb{C}$. If $f: \mathbb{R} \rightarrow \mathbb{R}$ is a $C^{0}$ solution of (1.4), then

$$
\begin{aligned}
f^{m}= & \frac{r_{1}^{m}}{\left(r_{1}-r_{2}\right)\left(r_{1}-r_{3}\right)}\left\{f^{2}(x)-\left(r_{2}+r_{3}\right) f(x)+r_{2} r_{3} x\right\} \\
& +\frac{r_{2}^{m}}{\left(r_{2}-r_{1}\right)\left(r_{2}-r_{3}\right)}\left\{f^{2}(x)-\left(r_{1}+r_{3}\right) f(x)+r_{1} r_{3} x\right\}
\end{aligned}
$$




$$
\begin{aligned}
+ & \frac{r_{3}^{m}}{\left(r_{3}-r_{1}\right)\left(r_{3}-r_{2}\right)}\left\{f^{2}(x)-\left(r_{1}+r_{2}\right) f(x)+r_{1} r_{2} x\right\} \\
f^{-m}(x)= & \frac{\left(\frac{1}{r_{1}}\right)^{m}}{\left(\frac{1}{r_{1}}-\frac{1}{r_{2}}\right)\left(\frac{1}{r_{1}}-\frac{1}{r_{3}}\right)}\left\{f^{-2}(x)-\left(\frac{1}{r_{2}}+\frac{1}{r_{3}}\right) f^{-1}(x)+\frac{1}{r_{2} r_{3}} x\right\} \\
& +\frac{\left(\frac{1}{r_{2}}\right)^{m}}{\left(\frac{1}{r_{2}}-\frac{1}{r_{1}}\right)\left(\frac{1}{r_{2}}-\frac{1}{r_{3}}\right)}\left\{f^{-2}(x)-\left(\frac{1}{r_{1}}+\frac{1}{r_{3}}\right) f^{-1}(x)+\frac{1}{r_{1} r_{3}} x\right\} \\
& +\frac{\left(\frac{1}{r_{3}}\right)^{m}}{\left(\frac{1}{r_{3}}-\frac{1}{r_{1}}\right)\left(\frac{1}{r_{3}}-\frac{1}{r_{2}}\right)}\left\{f^{-2}(x)-\left(\frac{1}{r_{1}}+\frac{1}{r_{2}}\right) f^{-1}(x)+\frac{1}{r_{1} r_{2}} x\right\}
\end{aligned}
$$

for any integer $m \geq 1$.

\section{Same signs with both expansion and contraction}

In this section we construct all $C^{0}$ solutions of (1.4) for the cases $\left(E_{+} C_{+}\right)$and $\left(E_{-} C_{-}\right)$listed in Table 1.

Lemma 3.1 Suppose that $0<r_{1}<1<r_{2}<r_{3}$. Then for $x_{0}=0$ and arbitrarily given $x_{1}, x_{2}$ such that

$$
x_{1}>0 \text { and }\left(r_{1}+r_{2}\right) x_{1}<x_{2}<\left(r_{1}+r_{3}\right) x_{1} \text {, }
$$

the sequence $\left(\ldots, x_{-2}, x_{-1} ; x_{0}, x_{1}, x_{2}, \ldots\right)$ defined by

$$
\begin{aligned}
x_{n+2}= & \left(r_{1}+r_{2}+r_{3}\right) x_{n+1}-\left(r_{1} r_{2}+r_{1} r_{3}+r_{2} r_{3}\right) x_{n}+r_{1} r_{2} r_{3} x_{n-1}, \\
x_{-n}= & \left(\frac{1}{r_{1}}+\frac{1}{r_{2}}+\frac{1}{r_{3}}\right) x_{-n+1}-\left(\frac{1}{r_{1} r_{2}}+\frac{1}{r_{1} r_{3}}+\frac{1}{r_{2} r_{3}}\right) x_{-n+2} \\
& +\frac{1}{r_{1} r_{2} r_{3}} x_{-n+3},
\end{aligned}
$$

is strictly increasing and satisfies

$$
\lim _{n \rightarrow+\infty} x_{n}=+\infty, \quad \lim _{n \rightarrow-\infty} x_{n}=-\infty
$$

Proof In order to prove the monotonicity and divergence of the sequence $\left(x_{n}\right)$, we prove that

$$
x_{n+1}>r_{2} x_{n}, \quad n=0,1,2, \ldots
$$

First of all, we claim that

$$
\left(r_{1}+r_{2}\right) x_{n+1}-r_{1} r_{2} x_{n}<x_{n+2}<\left(r_{2}+r_{3}\right) x_{n+1}-r_{2} r_{3} x_{n}, \quad n=0,1,2, \ldots
$$

Table 1 Three characteristic roots with same signs 
In fact (3.6) holds for $n=0$ by (3.1). Now, fix integer $k \geq 1$ and suppose that (3.6) holds for $n=k-1$. Then using (3.2) we get

$$
\begin{aligned}
& x_{k+2}-\left(r_{1}+r_{2}\right) x_{k+1}+r_{1} r_{2} x_{k} \\
& =r_{3} x_{k+1}-\left(r_{1} r_{3}+r_{2} r_{3}\right) x_{k}+r_{1} r_{2} r_{3} x_{k-1} \\
& \quad=r_{3}\left[x_{k+1}-\left(r_{1}+r_{2}\right) x_{k}+r_{1} r_{2} x_{k-1}\right] \\
& \quad>0, \\
& x_{k+2}-\left(r_{2}+r_{3}\right) x_{k+1}+r_{2} r_{3} x_{k} \\
& \quad=r_{1} x_{k+1}-\left(r_{1} r_{2}+r_{1} r_{3}\right) x_{k}+r_{1} r_{2} r_{3} x_{k-1} \\
& \quad=r_{1}\left[x_{k+1}-\left(r_{2}+r_{3}\right) x_{k}+r_{2} r_{3} x_{k-1}\right] \\
& \quad<0
\end{aligned}
$$

implying (3.6) holds for $n=k$. Thus (3.6) is proved by induction for $k$, from which we get

$$
\left(r_{1}+r_{2}\right) x_{n+1}-r_{1} r_{2} x_{n}<\left(r_{2}+r_{3}\right) x_{n+1}-r_{2} r_{3} x_{n}
$$

i.e., (3.5) holds. Since $r_{2}>1$, it follows that $\left(x_{n}\right)_{n \geq 0}$ is strictly increasing. From (3.5) we have

$$
x_{n+1}>r_{2} x_{n}>\cdots>r_{2}^{n} x_{1}>0 \text {. }
$$

Hence for arbitrarily given $M>0$, there exists an integer $N$ large enough such that

$$
x_{N+1}>r_{2}^{N} x_{1}>M>0 \text {. }
$$

Consequently, $\lim _{n \rightarrow+\infty} x_{n}=+\infty$. In a similar way as before, we get

$$
\left(\frac{1}{r_{1}}+\frac{1}{r_{2}}\right) x_{-n+1}-\frac{1}{r_{1} r_{2}} x_{-n+2}<x_{-n}<\left(\frac{1}{r_{1}}+\frac{1}{r_{3}}\right) x_{-n+1}-\frac{1}{r_{1} r_{3}} x_{-n+2}
$$

for $n=0,1,2, \ldots$. Hence $x_{-n+1}<\frac{1}{r_{1}} x_{-n+2}, n=0,1,2, \ldots$. This jointly with $x_{-1}<0$ implies $\left(x_{n}\right)_{n \leq 0}$ is strictly increasing and $\lim _{n \rightarrow-\infty} x_{n}=-\infty$.

Lemma 3.2 Suppose that $0<r_{1}<1<r_{2}<r_{3}$. Then for $x_{0}=0$ and arbitrary points $x_{1}, x_{2}$ such that

$$
x_{1}<0 \text { and }\left(r_{1}+r_{3}\right) x_{1}<x_{2}<\left(r_{1}+r_{2}\right) x_{1} \text {, }
$$

the sequence $\left(\ldots, x_{2}, x_{1} ; x_{0}, x_{-1}, x_{-2}, \ldots\right)$ defined by (3.2) and (3.3) is strictly decreasing and satisfies

$$
\lim _{n \rightarrow+\infty} x_{n}=-\infty, \quad \lim _{n \rightarrow-\infty} x_{n}=+\infty
$$


Proof Our strategy is similar to that of Lemma 3.1, in order to prove the monotonicity and divergence of the sequence $\left(x_{n}\right)$, we first show that

$$
x_{n+1}<r_{2} x_{n}, \quad n=0,1,2, \ldots
$$

By induction we can prove

$$
\left(r_{2}+r_{3}\right) x_{n+1}-r_{2} r_{3} x_{n}<x_{n+2}<\left(r_{1}+r_{2}\right) x_{n+1}-r_{1} r_{2} x_{n},
$$

which yields (3.13), whence

$$
\lim _{n \rightarrow+\infty} x_{n}=-\infty
$$

On the other hand, by a similar procedure we inductively prove that

$$
\left(\frac{1}{r_{1}}+\frac{1}{r_{3}}\right) x_{-n+1}-\frac{1}{r_{1} r_{3}} x_{-n+2}<x_{-n}<\left(\frac{1}{r_{1}}+\frac{1}{r_{2}}\right) x_{-n+1}-\frac{1}{r_{1} r_{2}} x_{-n+2}
$$

for $n=0,1, \ldots$, which yields

$$
x_{-n+1}>\frac{1}{r_{1}} x_{-n+2},
$$

implying

$$
\lim _{n \rightarrow-\infty} x_{n}=+\infty
$$

This completes the proof.

Theorem 3.1 Suppose that $0<r_{1}<1<r_{2}<r_{3}$. Then all $C^{0}$ solutions $f: \mathbb{R} \rightarrow \mathbb{R}$ of $(1.4)$ are strictly increasing. Additionally:

(i) Iff has fixed points, then 0 is the unique fixed point and

$$
f(x)=\left\{\begin{array}{ll}
f_{i}(x), & x \geq 0, \\
f_{j}(x), & x<0,
\end{array} \quad i, j=1,2,\right.
$$

where $f_{1}(x)=r_{1} x$ and $f_{2}(x)$ is a solution given in Theorem 1 of $[14]$.

(ii) If $f(x)>x$ for all $x \in \mathbb{R}$, then the set of $f$ contains both $f(x)>\max \left\{r_{1} x, r_{i} x\right\}(i=2,3)$ constructed by Theorem 2 of [14] and

$$
f(x):= \begin{cases}f_{n}(x), & x \in\left[x_{n}, x_{n+1}\right], n=0,1, \ldots, \\ f_{-n}^{-1}(x), & x \in\left[x_{-n}, x_{-n+1}\right], n=1,2, \ldots\end{cases}
$$

where the bilateral sequence $\left(x_{i}\right)$ is given in Lemma 3.1, and $f_{n}:\left[x_{n}, x_{n+1}\right] \rightarrow\left[x_{n+1}, x_{n+2}\right]$ and $f_{-n}:\left[x_{-n+1}, x_{-n+2}\right] \rightarrow\left[x_{-n}, x_{-n+1}\right]$ are orientation-preserving homeomorphisms defined inductively as

$$
\begin{aligned}
& f_{n+2}(x)=\left(r_{1}+r_{2}+r_{3}\right) x-\left(r_{1} r_{2}+r_{1} r_{3}+r_{2} r_{3}\right) f_{n+1}^{-1}(x)+r_{1} r_{2} r_{3} f_{n}^{-1}\left(f_{n+1}^{-1}(x)\right), \\
& f_{-n}(x)=\left(\frac{1}{r_{1}}+\frac{1}{r_{2}}+\frac{1}{r_{3}}\right) x-\left(\frac{1}{r_{1} r_{2}}+\frac{1}{r_{1} r_{3}}+\frac{1}{r_{2} r_{3}}\right) f_{-n+1}^{-1}(x)+\frac{1}{r_{1} r_{2} r_{3}} f_{-n+2}^{-1}\left(f_{-n+1}^{-1}(x)\right),
\end{aligned}
$$


which are uniquely determined by two given functions $f_{0}:\left[x_{0}, x_{1}\right] \rightarrow\left[x_{1}, x_{2}\right]$ and $f_{1}$ : $\left[x_{1}, x_{2}\right] \rightarrow\left[x_{2}, x_{3}\right]$.

(iii) If $f(x)<x$ for all $x \in \mathbb{R}$, then the set of $f$ contains both $f(x)<\max \left\{r_{1} x, r_{i} x\right\}(i=2,3)$ constructed by Theorem 2 of [14] and

$$
f(x):= \begin{cases}f_{n}(x), & x \in\left[x_{n+1}, x_{n}\right], n=0,1, \ldots \\ f_{-n}^{-1}(x), & x \in\left[x_{-n+1}, x_{-n}\right], n=1,2, \ldots\end{cases}
$$

where the bilateral sequence $\left(x_{i}\right)$ is given in Lemma 3.2, and $f_{n}:\left[x_{n+1}, x_{n}\right] \rightarrow\left[x_{n+2}, x_{n+1}\right]$ and $f_{-n}:\left[x_{-n+2}, x_{-n+1}\right] \rightarrow\left[x_{-n+1}, x_{-n}\right]$ are orientation-preserving homeomorphisms defined inductively as (3.18) which are uniquely determined by two given functions $f_{0}:\left[x_{1}, x_{0}\right] \rightarrow$ $\left[x_{2}, x_{1}\right]$ and $f_{1}:\left[x_{2}, x_{1}\right] \rightarrow\left[x_{3}, x_{2}\right]$.

Proof For an indirect proof, assume that $f$ is a $C^{0}$ strictly decreasing solution of (1.4). By Lemma 2.6 we get

$$
\lim _{m \rightarrow \infty} \frac{f^{m}}{r_{3}^{m}}=\frac{1}{\left(r_{3}-r_{2}\right)\left(r_{3}-r_{1}\right)}\left\{f^{2}(x)-\left(r_{1}+r_{2}\right) f(x)+r_{1} r_{2} x\right\}
$$

for any integer $m \geq 1$. The function $\lim _{m \rightarrow \infty} f^{m} / r_{3}^{m}$ is both nondecreasing for even $m$ and nonincreasing for odd $m$, there is a constant $c_{1}$ such that

$$
f^{2}(x)-\left(r_{1}+r_{2}\right) f(x)+r_{1} r_{2} x=c_{1}
$$

for all $x \in \mathbb{R}$. Substituting (3.21) into (1.4), we get $c_{1}=r_{3} c_{1}$ implying $c_{1}=0$ since $r_{3} \neq 1$. Hence (3.21) reduces to the equation

$$
f^{2}(x)-\left(r_{1}+r_{2}\right) f(x)+r_{1} r_{2} x=0,
$$

which has only strictly increasing solutions by Theorem 2 from [14]; a contradiction.

In order to prove the case (i), let $f$ be a $C^{0}$ strictly increasing solution with fixed points. Then $f$ has the unique fixed point 0 by Lemma 2.4, which implies either $0<f(x)<x$ or $f(x)>x$ for $x>0$. In the case $0<f(x)<x$ we have $\lim _{m \rightarrow \infty} f^{m}(x)=0$ because 0 is an attracting fixed point, then (3.22) from (3.20) directly. Thus (3.22) yields $f(x)=r_{1} x$ by Theorem 2 of [14], which is the $C^{0}$ strictly increasing solution satisfying $0<f(x)<x$ for $x>0$. The other case $f(x)>x$ can be reduced to the first one by considering the dual equation (1.5). Using Lemma 2.6 and repeating the same method as the previous one to eliminate $1 / r_{1}$ we have

$$
f^{-2}(x)-\left(\frac{1}{r_{2}}+\frac{1}{r_{3}}\right) f^{-1}+\frac{1}{r_{2} r_{3}} x=0, \quad x>0,
$$

that is, $f$ is a solution of the equation

$$
f^{2}(x)-\left(r_{2}+r_{3}\right) f(x)-r_{2} r_{3} x=0, \quad x>0 .
$$

Thus $f$ is a solution given in Theorem 1 of $[14]$ on $(0, \infty)$. A similar discussion can be done for the half-line $(-\infty, 0)$. Therefore, every $C^{0}$ solution $f$ of $(1.4)$ involving a fixed point has the form (3.16). 
In order to prove (ii), we use the formula

$$
\begin{aligned}
f^{m+1}-f^{m}= & \frac{r_{1}^{m}\left(r_{1}-1\right)}{\left(r_{1}-r_{2}\right)\left(r_{1}-r_{3}\right)}\left\{f^{2}(x)-\left(r_{2}+r_{3}\right) f(x)+r_{2} r_{3} x\right\} \\
& +\frac{r_{2}^{m}\left(r_{2}-1\right)}{\left(r_{2}-r_{1}\right)\left(r_{2}-r_{3}\right)}\left\{f^{2}(x)-\left(r_{1}+r_{3}\right) f(x)+r_{1} r_{3} x\right\} \\
& +\frac{r_{3}^{m}\left(r_{3}-1\right)}{\left(r_{3}-r_{1}\right)\left(r_{3}-r_{2}\right)}\left\{f^{2}(x)-\left(r_{1}+r_{2}\right) f(x)+r_{1} r_{2} x\right\},
\end{aligned}
$$

generated by Lemma 2.6. If $f(x)>x$ for all $x \in \mathbb{R}$, we assert that one of the three situations holds:

$$
\begin{aligned}
& \left\{\begin{array}{l}
I_{r_{1}, m}:=\frac{r_{1}^{m}\left(r_{1}-1\right)}{\left(r_{1}-r_{2}\right)\left(r_{1}-r_{3}\right)}\left\{f^{2}(x)-\left(r_{2}+r_{3}\right) f(x)+r_{2} r_{3} x\right\}>0, \\
I_{r_{2}, m}:=\frac{r_{2}^{m}\left(r_{2}-1\right)}{\left(r_{2}-r_{1}\right)\left(r_{2}-r_{3}\right)}\left\{f^{2}(x)-\left(r_{1}+r_{3}\right) f(x)+r_{1} r_{3} x\right\}>0, \\
I_{r_{3}, m}:=\frac{r_{3}^{m}\left(r_{3}-1\right)}{\left(r_{3}-r_{1}\right)\left(r_{3}-r_{2}\right)}\left\{f^{2}(x)-\left(r_{1}+r_{2}\right) f(x)+r_{1} r_{2} x\right\}>0,
\end{array}\right. \\
& f^{2}(x)-\left(r_{1}+r_{2}\right) f(x)+r_{1} r_{2} x=0, \\
& f^{2}(x)-\left(r_{1}+r_{3}\right) f(x)+r_{1} r_{3} x=0 .
\end{aligned}
$$

Clearly, $f(x)>x$ implies that $f^{m+1}(x)>f^{m}(x)$ and $f^{-m}(x)>f^{-m-1}(x)$. Firstly, from $0<r_{1}<1<$ $r_{2}<r_{3}$ the sign of $f^{m+1}-f^{m}$ is uniquely determined by the third term in (3.24) when $m$ is large enough, that is, $I_{r_{3}, m} \geq 0$, thus we get the third inequality in (3.25) or the equality (3.26). Secondly, by the same argument for the dual equation (1.5), we get $I_{r_{1}, m} \geq 0$ using $f^{-m}(x)>f^{-m-1}(x)$. Further, we prove that $I_{r_{1}, m} \neq 0$, otherwise, the equality $f^{2}(x)-\left(r_{2}+\right.$ $\left.r_{3}\right) f(x)+r_{2} r_{3}=0$ implies $f$ has the fixed point 0 by Theorem 1 of [14]. This contradicts the assumption $f(x)>x$, implying the first inequality of (3.25) holds, i.e., $I_{r_{1}, m}>0$. Finally, we prove $I_{r_{2}, m} \geq 0$. Conversely, assume that $I_{r_{2}, m}<0$. When (3.26) holds we get $f^{m+1}(x)<f^{m}(x)$ when $m$ is large enough by using (3.24), which contradicts $f(x)>x$ on $\mathbb{R}$. Thus the second inequality in (3.25) and the equality (3.27) are proved.

In addition, we see that (3.25) is equivalent to

$$
\left\{\begin{array}{l}
f^{2}(x)-\left(r_{2}+r_{3}\right) f(x)+r_{2} r_{3} x<0 \\
f^{2}(x)-\left(r_{1}+r_{3}\right) f(x)+r_{1} r_{3} x<0 \\
f^{2}(x)-\left(r_{1}+r_{2}\right) f(x)+r_{1} r_{2} x>0
\end{array}\right.
$$

implying

$$
\left\{\begin{array}{l}
\left(r_{1}+r_{2}\right) f(x)-r_{1} r_{2} x<f^{2}(x)<\left(r_{2}+r_{3}\right) f(x)-r_{2} r_{3} x, \\
\left(r_{1}+r_{2}\right) f(x)-r_{1} r_{2} x<f^{2}(x)<\left(r_{1}+r_{3}\right) f(x)-r_{1} r_{3} x
\end{array}\right.
$$

that is,

$$
\left(r_{1}+r_{2}\right) f(x)-r_{1} r_{2} x<f^{2}(x)<\left(r_{1}+r_{3}\right) f(x)-r_{1} r_{3} x, \quad \forall x \in \mathbb{R} .
$$

In a word, if $f(x)>x$ for all $x \in \mathbb{R}$, then $f$ satisfies the inequality (3.28) or the equality (3.26) or (3.27). 
Conversely, all solutions of (3.26) satisfying $f(x)>\max \left\{r_{1} x, r_{2} x\right\}$ and all solutions of (3.27) satisfying $f(x)>\max \left\{r_{1} x, r_{3} x\right\}$, constructed by Theorem 2 of [14], are $C^{0}$ strictly increasing solutions of (1.4) satisfying $f(x)>x$ for all $x \in \mathbb{R}$. Moreover, every $C^{0}$ strictly increasing solution $f$ of (1.4) fulfilling (3.28) is uniquely determined up to two orientation-preserving homeomorphisms $f_{0}:\left[x_{0}, x_{1}\right] \rightarrow\left[x_{1}, x_{2}\right]$ and $f_{1}:\left[x_{1}, x_{2}\right] \rightarrow\left[x_{2}, x_{3}\right]$ and can be constructed by the recursively orientation-preserving homeomorphisms

$$
\begin{aligned}
& f_{n}:\left[x_{n}, x_{n+1}\right] \rightarrow\left[x_{n+1}, x_{n+2}\right], \quad n=0,1,2, \ldots, \\
& f_{-n}:\left[x_{-n+1}, x_{-n+2}\right] \rightarrow\left[x_{-n}, x_{-n+1}\right], \quad n=1,2, \ldots
\end{aligned}
$$

defined by (3.18), where the sequence $\left(\ldots, x_{-2}, x_{-1} ; x_{0}, x_{1}, x_{2}, \ldots\right)$ is given in Lemma 3.1. Actually, by induction the inequality (3.6) and the orientation-preserving homeomorphisms (3.29) yield

$$
\left(r_{1}+r_{2}\right) f_{n}(x)-r_{1} r_{2} x<f_{n+1} \circ f_{n}(x)<\left(r_{2}+r_{3}\right) f_{n}(x)-r_{2} r_{3} x
$$

for arbitrary $x \in\left[x_{n}, x_{n+1}\right], n=0,1, \ldots$, then the function $f$ defined by those orientationpreserving homeomorphisms $f_{n}$ is the unique solution of $(1.4)$ on $[0,+\infty)$. On the other hand, the inequality (3.10) and the orientation-preserving homeomorphisms (3.30) yield

$$
\left(\frac{1}{r_{1}}+\frac{1}{r_{2}}\right) f_{-n}(x)-\frac{1}{r_{1} r_{2}} x<f_{-n-1} \circ f_{-n}(x)<\left(\frac{1}{r_{1}}+\frac{1}{r_{3}}\right) f_{-n}(x)-\frac{1}{r_{1} r_{3}} x
$$

for arbitrary $x \in\left[x_{-n+1}, x_{-n+2}\right], n=1,2, \ldots$, then the function $f^{-1}$ defined by those orientation-preserving homeomorphisms $f_{-n}$ is the unique solution of the dual equation (1.5) on $(-\infty, 0]$. Finally, we define $f$ as (3.17), i.e.,

$$
f(x):= \begin{cases}f_{n}(x), & x \in\left[x_{n}, x_{n+1}\right], n=0,1, \ldots \\ f_{-n}^{-1}(x), & x \in\left[x_{-n}, x_{-n+1}\right], n=1,2, \ldots\end{cases}
$$

Then $f: \mathbb{R} \rightarrow \mathbb{R}$ is the unique $C^{0}$ strictly increasing solution of (1.4), depending on the given initial functions $f_{0}$ and $f_{1}$. Thus all those $C^{0}$ strictly increasing solutions $f$ of (1.4), fulfilling inequality (3.28), can be constructed by this method.

Using the same idea and arguments we prove the case (iii). From $f(x)<x$ we see that $f^{m+1}(x)<f^{m}(x)$ for $x \in \mathbb{R}$. By using (3.24) and a similar discussion to (ii), $f(x)<x$ shows that one of three situations holds: (3.26) and (3.27), and

$$
\left\{\begin{array}{l}
I_{r_{1}, m}:=\frac{r_{1}^{m}\left(r_{1}-1\right)}{\left(r_{1}-r_{2}\right)\left(r_{1}-r_{3}\right)}\left\{f^{2}(x)-\left(r_{2}+r_{3}\right) f(x)+r_{2} r_{3} x\right\}<0, \\
I_{r_{2}, m}:=\frac{r_{2}^{m}\left(r_{2}-1\right)}{\left(r_{2}-r_{1}\right)\left(r_{2}-r_{3}\right)}\left\{f^{2}(x)-\left(r_{1}+r_{3}\right) f(x)+r_{1} r_{3} x\right\}<0, \\
I_{r_{3}, m}:=\frac{r_{3}^{m}\left(r_{3}-1\right)}{\left(r_{3}-r_{1}\right)\left(r_{3}-r_{2}\right)}\left\{f^{2}(x)-\left(r_{1}+r_{2}\right) f(x)+r_{1} r_{2} x\right\}<0,
\end{array}\right.
$$

i.e.,

$$
\left(r_{2}+r_{3}\right) f(x)-r_{2} r_{3} x<f^{2}(x)<\left(r_{1}+r_{2}\right) f(x)-r_{1} r_{2} x, \quad \forall x \in \mathbb{R} .
$$

Conversely, all solutions of (3.26) satisfying $f(x)<\max \left\{r_{1} x, r_{2} x\right\}$ and all solutions of (3.27) satisfying $f(x)<\max \left\{r_{1} x, r_{3} x\right\}$, constructed by Theorem 2 of [14], are $C^{0}$ strictly increasing 
solutions of (1.4) satisfying $f(x)<x$ for all $x \in \mathbb{R}$. Furthermore, every $C^{0}$ strictly increasing solution $f$ of (1.4) fulfilling (3.31) is uniquely determined up to two orientation-preserving homeomorphisms $f_{0}:\left[x_{0}, x_{1}\right] \rightarrow\left[x_{1}, x_{2}\right]$ and $f_{1}:\left[x_{1}, x_{2}\right] \rightarrow\left[x_{2}, x_{3}\right]$ and can be constructed by the recursively orientation-preserving homeomorphisms

$$
\begin{aligned}
& f_{n}:\left[x_{n+1}, x_{n}\right] \rightarrow\left[x_{n+2}, x_{n+1}\right], \quad n=0,1,2, \ldots, \\
& f_{-n}:\left[x_{-n+2}, x_{-n+1}\right] \rightarrow\left[x_{-n+1}, x_{-n}\right], \quad n=1,2, \ldots,
\end{aligned}
$$

defined by (3.19), where the sequence $\left(\ldots, x_{-2}, x_{-1} ; x_{0}, x_{1}, x_{2}, \ldots\right)$ is given in Lemma 3.2. In fact, the inequality (3.14) and the orientation-preserving homeomorphisms (3.32) yield

$$
\left(r_{2}+r_{3}\right) f_{n}(x)-r_{2} r_{3} x<f_{n+1} \circ f_{n}(x)<\left(r_{1}+r_{2}\right) f_{n}(x)-r_{1} r_{2} x
$$

for arbitrary $x \in\left[x_{n+1}, x_{n}\right], n=0,1, \ldots$, then the function $f$ defined by those orientationpreserving homeomorphisms $f_{n}$ is the unique solution of $(1.4)$ on $(-\infty, 0]$. On the other hand, (3.15) and the orientation-preserving homeomorphisms (3.33) yield

$$
\left(\frac{1}{r_{1}}+\frac{1}{r_{3}}\right) f_{-n}(x)-\frac{1}{r_{1} r_{3}} x<f_{-n-1} \circ f_{-n}(x)<\left(\frac{1}{r_{1}}+\frac{1}{r_{2}}\right) f_{-n}(x)-\frac{1}{r_{1} r_{2}} x
$$

for arbitrary $x \in\left[x_{-n+2}, x_{-n+1}\right], n=1,2, \ldots$, then the function $f^{-1}$ defined by those orientation-preserving homeomorphisms $f_{-n}$ is the unique solution of the dual equation of (1.5) on $[0,+\infty)$. Finally, we see that the unique $C^{0}$ strictly increasing solution of (1.4), depending on the given initial functions $f_{0}$ and $f_{1}$, can be presented in the form

$$
f(x):= \begin{cases}f_{n}(x), & x \in\left[x_{n+1}, x_{n}\right], n=0,1, \ldots, \\ f_{-n}^{-1}(x), & x \in\left[x_{-n+1}, x_{-n}\right], n=1,2, \ldots\end{cases}
$$

All those $C^{0}$ strictly increasing solutions $f$ of (1.4), fulfilling inequality (3.31), can be constructed by this method. This completes the proof.

Corollary 3.1 Suppose that $0<r_{3}<r_{2}<1<r_{1}$. Then every $C^{0}$ solution $f: \mathbb{R} \rightarrow \mathbb{R}$ of (1.4) is strictly increasing and $f^{-1}$ is a solution given in Theorem 3.1.

Theorem 3.2 Suppose that $r_{1}<r_{2}<-1<r_{3}<0$. Then all $C^{0}$ solutions $f: \mathbb{R} \rightarrow \mathbb{R}$ of (1.4) are strictly decreasing and $x=0$ is the unique fixed point of every $f$. Moreover:

(i) If $x=0$ is attractive fixed point of $f^{2}$, then $f(x)=r_{3} x$.

(ii) If $x=0$ is repelling fixed point of $f^{2}$, then $f(x)$ is a solution in the class given in Theorem 3 of [14].

Proof For an indirect proof, assume that $f: \mathbb{R} \rightarrow \mathbb{R}$ is a $C^{0}$ strictly increasing solution of (1.4). By using (2.6) and similar arguments to the preceding part of Theorem 3.1, we get at last

$$
f^{2}(x)-\left(r_{2}+r_{3}\right) f(x)+r_{2} r_{3} x=0,
$$

which yields $f_{1}(x)=r_{2} x$ and $f_{2}(x)=r_{3} x$ by Theorem 5 of [14]. This contradicts the strictly increasing of $f$, then every $C^{0}$ solution of (1.4) is strictly decreasing. It is easy to see that 
$x=0$ is the unique fixed point of every $C^{0}$ strictly decreasing solution by Lemma 2.4 and $f^{2}$ is $C^{0}$ strictly increasing satisfying $f^{2}(0)=0$. It follows that either $\lim _{m \rightarrow+\infty} f^{2 m}(x)=0$ (i.e., 0 is an attractive fixed point of $f^{2}$ ) or $\lim _{m \rightarrow+\infty} f^{2 m}(x)=\infty$ (i.e., 0 is a repelling fixed point of $\left.f^{2}\right)$.

In case (i), $x=0$ is an attractive fixed point of $f^{2}$. Using Lemma 2.6 we have

$$
\lim _{m \rightarrow \infty} \frac{f^{2 m}}{r_{1}^{2 m}}=\frac{1}{\left(r_{1}-r_{2}\right)\left(r_{1}-r_{3}\right)}\left\{f^{2}(x)-\left(r_{2}+r_{3}\right) f(x)+r_{2} r_{3} x\right\} .
$$

Since the left-hand side tends to 0 as $n \rightarrow \infty$ we have

$$
f^{2}(x)-\left(r_{2}+r_{3}\right) f(x)+r_{2} r_{3} x=0
$$

which yields $f_{1}(x)=r_{2} x$ and $f_{2}(x)=r_{3} x$ by Theorem 5 of [14]. Thus $f_{2}(x)=r_{3} x$ is the only solution of (1.4), where $f^{2}$ has the attractive fixed point $x=0$.

In case (ii), since $x=0$ is a repelling fixed point of $f^{2}$, it is an attractive fixed point of $f^{-2}$. Using Lemma 2.6 we get

$$
\begin{aligned}
f^{-2 m}(x)= & \frac{\left(\frac{1}{r_{1}}\right)^{2 m}}{\left(\frac{1}{r_{1}}-\frac{1}{r_{2}}\right)\left(\frac{1}{r_{1}}-\frac{1}{r_{3}}\right)}\left\{f^{-2}(x)-\left(\frac{1}{r_{2}}+\frac{1}{r_{3}}\right) f^{-1}(x)+\frac{1}{r_{2} r_{3}} x\right\} \\
& +\frac{\left(\frac{1}{r_{2}}\right)^{2 m}}{\left(\frac{1}{r_{2}}-\frac{1}{r_{1}}\right)\left(\frac{1}{r_{2}}-\frac{1}{r_{3}}\right)}\left\{f^{-2}(x)-\left(\frac{1}{r_{1}}+\frac{1}{r_{3}}\right) f^{-1}(x)+\frac{1}{r_{1} r_{3}} x\right\} \\
& +\frac{\left(\frac{1}{r_{3}}\right)^{2 m}}{\left(\frac{1}{r_{3}}-\frac{1}{r_{1}}\right)\left(\frac{1}{r_{3}}-\frac{1}{r_{2}}\right)}\left\{f^{-2}(x)-\left(\frac{1}{r_{1}}+\frac{1}{r_{2}}\right) f^{-1}(x)+\frac{1}{r_{1} r_{2}} x\right\} .
\end{aligned}
$$

By the same argument as (i) to remove $1 / r_{3}$, we have

$$
f^{-2}(x)-\left(\frac{1}{r_{1}}+\frac{1}{r_{2}}\right) f^{-1}(x)+\frac{1}{r_{1} r_{2}} x=0 .
$$

It follows that

$$
f^{2}(x)-\left(r_{1}+r_{2}\right) f(x)+r_{1} r_{2} x=0,
$$

implying $f(x)$ is a solution given in Theorem 3 of [14]. This completes the proof.

Corollary 3.2 Suppose that $r_{3}<-1<r_{2}<r_{1}<0$. Then all $C^{0}$ solutions $f: \mathbb{R} \rightarrow \mathbb{R}$ of (1.4) are strictly decreasing with the unique fixed point 0 , and the inverse $f^{-1}$ is a solution given in Theorem 3.2.

\section{Different signs with both expansion and contraction}

In this section we construct all $C^{0}$ solutions of (1.4) for the cases $\left(E_{+} C_{-}\right),\left(E_{-} C_{+}\right),\left(C_{+} C_{-}\right)$, and $\left(E_{+} E_{-}\right)$, where Table 2 contains one negative root and two positive ones, Table 3 contains two negative roots and one positive root.

Theorem 4.1 Suppose that $1<-r_{1}<r_{2}<r_{3}\left(1<r_{2}<-r_{1}<r_{3}, 1<r_{2}<r_{3}<-r_{1}, 0<-r_{1}<1<\right.$ $\left.r_{2}<r_{3}\right)$. 
Table 2 Two positive and one negative characteristic roots

\begin{tabular}{lll}
\hline $1<-r_{1}<r_{2}<r_{3}$ & $1<r_{2}<-r_{1}<r_{3}$ & $1<r_{2}<r_{3}<-r_{1}$ \\
$0<-r_{1}<r_{2}<r_{3}<1$ & $0<r_{2}<-r_{1}<r_{3}<1$ & $0<r_{2}<r_{3}<-r_{1}<1$ \\
$0<-r_{1}<1<r_{2}<r_{3}$ & $0<r_{2}<1<-r_{1}<r_{3}$ & $0<r_{2}<1<r_{3}<-r_{1}$ \\
$0<r_{2}<r_{3}<1<-r_{1}$ & $0<r_{2}<-r_{1}<1<r_{3}$ & $0<-r_{1}<r_{2}<1<r_{3}$ \\
\hline
\end{tabular}

Table 3 One positive and two negative characteristic roots

\begin{tabular}{lll}
\hline $1<-r_{1}<-r_{2}<r_{3}$ & $1<-r_{1}<r_{3}<-r_{2}$ & $1<r_{3}<-r_{1}<-r_{2}$ \\
$0<-r_{1}<-r_{2}<r_{3}<1$ & $0<-r_{1}<r_{3}<-r_{2}<1$ & $0<r_{3}<-r_{1}<-r_{2}<1$ \\
$0<-r_{1}<1<-r_{2}<r_{3}$ & $0<-r_{1}<1<r_{3}<-r_{2}$ & $0<r_{3}<1<-r_{1}<-r_{2}$ \\
$0<r_{3}<-r_{1}<1<-r_{2}$ & $0<-r_{1}<r_{3}<1<-r_{2}$ & $0<-r_{1}<-r_{2}<1<r_{3}$ \\
\hline
\end{tabular}

(i) If $: \mathbb{R} \rightarrow \mathbb{R}$ is a $C^{0}$ strictly increasing solution of (1.4), then $f$ is a function in the class given in Theorem 1 of [14].

(ii) If $: \mathbb{R} \rightarrow \mathbb{R}$ is a $C^{0}$ strictly decreasing solution of $(1.4)$, then $f(x)=r_{1} x$.

Proof We first consider the case $1<-r_{1}<r_{2}<r_{3}$. Let $f: \mathbb{R} \rightarrow \mathbb{R}$ be a $C^{0}$ strictly increasing solution of (1.4), then $f^{-1}$ is a $C^{0}$ strictly increasing solution of the dual equation (1.5). Making use of Lemma 2.6 and the monotonicity of $f^{-1}$ to remove $1 / r_{1}$, we get

$$
f^{-2}(x)-\left(\frac{1}{r_{2}}+\frac{1}{r_{3}}\right) f^{-1}(x)+\frac{1}{r_{2} r_{3}} x=0,
$$

that is,

$$
f^{2}(x)-\left(r_{2}+r_{3}\right) f(x)+r_{2} r_{3} x=0
$$

thus $f$ is a function in the class given in Theorem 1 of [14]. On the other hand, if $f: \mathbb{R} \rightarrow \mathbb{R}$ is a $C^{0}$ strictly decreasing solution of (1.4), by the same method as previous to remove $r_{3}$ of (1.4), the unique $C^{0}$ strictly decreasing solution $f(x)=r_{1} x$ is obtained by Theorem 5 of [14].

In what follows attention is paid to the case $1<r_{2}<-r_{1}<r_{3}$. If $f: \mathbb{R} \rightarrow \mathbb{R}$ is a $C^{0}$ strictly increasing solution of (1.4), from Lemma 2.6 and the monotonicity of $f$ we have

$$
\begin{aligned}
\lim _{m \rightarrow+\infty} \frac{f^{m}}{r_{1}^{m}}= & \frac{1}{\left(r_{1}-r_{2}\right)\left(r_{1}-r_{3}\right)}\left\{f^{2}(x)-\left(r_{2}+r_{3}\right) f(x)+r_{2} r_{3} x\right\} \\
& +\lim _{m \rightarrow+\infty} \frac{\left(\frac{r_{3}}{r_{1}}\right)^{m}}{\left(r_{3}-r_{1}\right)\left(r_{3}-r_{2}\right)}\left\{f^{2}(x)-\left(r_{1}+r_{2}\right) f(x)+r_{1} r_{2} x\right\} .
\end{aligned}
$$

Note that $\lim _{m \rightarrow+\infty} f^{m} / r_{1}^{m}$ is nondecreasing for even $m$ and nonincreasing for odd $m$, the left-hand side of (4.1) is a constant. We assert that

$$
f^{2}(x)-\left(r_{2}+r_{3}\right) f(x)+r_{2} r_{3} x=0
$$

Otherwise, $f^{2}(x)-\left(r_{2}+r_{3}\right) f(x)+r_{2} r_{3} x \neq 0$ yields $f(x) \neq r_{2} x$, then we have $f^{2}(x)-\left(r_{1}+\right.$ $\left.r_{2}\right) f(x)+r_{1} r_{2} x \neq 0$ by Theorem 5 of [14]. Consequently, the limit of the right-hand side of (4.1) tends to infinity as $m \rightarrow \infty$ but the left-hand side of (4.1) is constant. The contradiction illustrates (4.2). So every $C^{0}$ strictly increasing solution $f$ of (1.4) is a function in the 
class given in Theorem 1 of [14]. On the other hand, if $f: \mathbb{R} \rightarrow \mathbb{R}$ is a $C^{0}$ strictly decreasing solution of (1.4), the conclusion can be obtained by a similar argument to the first case $1<-r_{1}<r_{2}<r_{3}$.

For the case $1<r_{2}<r_{3}<-r_{1}$, assume that $f: \mathbb{R} \rightarrow \mathbb{R}$ is a $C^{0}$ strictly increasing solution of (1.4). Using Lemma 2.6 and the monotonicity of $f$ to remove $r_{1}$, we obtain

$$
f^{2}(x)-\left(r_{2}+r_{3}\right) f(x)+r_{2} r_{3} x=0
$$

implying that $f$ is a function in the class given in Theorem 1 of [14]. On the other hand, if $f: \mathbb{R} \rightarrow \mathbb{R}$ is a $C^{0}$ strictly decreasing solution of (1.4), we consider the dual equation (1.5). By Lemma 2.6 and the monotonicity of $f^{-1}$ to eliminate $1 / r_{2}$, we eventually have

$$
f^{2}(x)-\left(r_{1}+r_{3}\right) f(x)+r_{1} r_{3} x=0 .
$$

Thus $f(x)=r_{1} x$ is the only $C^{0}$ strictly decreasing solution according to Theorem 5 of [14].

The proof for the case $0<-r_{1}<1<r_{2}<r_{3}$ is the same as that of the case $1<-r_{1}<r_{2}<r_{3}$ and is omitted. This completes the proof.

By considering the dual equation (1.5) we get the following corollary.

Corollary 4.1 Suppose that $0<-r_{1}<r_{2}<r_{3}<1\left(0<r_{2}<-r_{1}<r_{3}<1,0<r_{2}<r_{3}<-r_{1}<1\right.$, $\left.0<r_{2}<r_{3}<1<-r_{1}\right)$.Iff $: \mathbb{R} \rightarrow \mathbb{R}$ is a $C^{0}$ solution of $(1.4)$, then $f^{-1}$ is a function in the class given in Theorem 4.1.

Theorem 4.2 Suppose that $0<-r_{1}<r_{2}<1<r_{3}\left(0<r_{2}<-r_{1}<1<r_{3}\right)$.

(i) If $: \mathbb{R} \rightarrow \mathbb{R}$ is a $C^{0}$ strictly increasing solution of (1.4), then $f$ is a function in the class given in Theorem 2 of [14].

(ii) Iff $: \mathbb{R} \rightarrow \mathbb{R}$ is a $C^{0}$ strictly decreasing solution of $(1.4)$, then $f(x)=r_{1} x$.

The proof is similar to that of the case $1<-r_{1}<r_{2}<r_{3}\left(1<r_{2}<-r_{1}<r_{3}\right)$ in Theorem 4.1 and is omitted. By using the dual equation (1.5) we have the following corollary.

Corollary 4.2 Suppose that $0<r_{2}<1<r_{3}<-r_{1}\left(0<r_{2}<1<-r_{1}<r_{3}\right)$. Iff $: \mathbb{R} \rightarrow \mathbb{R}$ is a $C^{0}$ solution of (1.4), then $f^{-1}$ is a function in the class given in Theorem 4.2.

Theorem 4.3 Suppose that $1<-r_{1}<-r_{2}<r_{3}\left(1<-r_{1}<r_{3}<-r_{2}, 1<r_{3}<-r_{1}<-r_{2}, 0<r_{3}<\right.$ $\left.1<-r_{1}<-r_{2}\right)$.

(i) Iff $: \mathbb{R} \rightarrow \mathbb{R}$ is a $C^{0}$ strictly increasing solution of $(1.4)$, then $f(x)=r_{3} x$.

(ii) If $f: \mathbb{R} \rightarrow \mathbb{R}$ is a $C^{0}$ strictly decreasing solution of (1.4), then $f$ is a function in the class given in Theorem 3 of [14].

Proof First we consider the case $1<-r_{1}<-r_{2}<r_{3}$. Let $f: \mathbb{R} \rightarrow \mathbb{R}$ be a $C^{0}$ strictly increasing solution of (1.4), then $f^{-1}$ is a $C^{0}$ strictly increasing solution of the dual equation (1.5). Making use of Lemma 2.6 and the monotonicity of $f^{-1}$ to eliminate $1 / r_{1}$, we get the unique $C^{0}$ strictly increasing solution $f(x)=r_{3} x$ according to Theorem 5 of [14]. Assume that $f: \mathbb{R} \rightarrow \mathbb{R}$ is a $C^{0}$ strictly decreasing solution of (1.4). Removing $r_{3}$ by Lemma 2.6 and the 
monotonicity of $f$ we have

$$
f^{2}(x)-\left(r_{1}+r_{2}\right) f(x)+r_{1} r_{2} x=0
$$

naturally, $f$ is a function in the class given in Theorem 3 of [14].

For the case $1<-r_{1}<r_{3}<-r_{2}$, assume that $f: \mathbb{R} \rightarrow \mathbb{R}$ is a $C^{0}$ strictly increasing solution of (1.4). By removing $r_{2}$, we get

$$
f^{2}(x)-\left(r_{1}+r_{3}\right) f(x)+r_{1} r_{3} x=0
$$

then the unique $C^{0}$ strictly increasing solution $f(x)=r_{3} x$ is obtained by Theorem 5 of [14]. Assume that $f: \mathbb{R} \rightarrow \mathbb{R}$ is a $C^{0}$ strictly decreasing solution of (1.4). By Lemma 2.6 we have

$$
\begin{aligned}
\lim _{m \rightarrow+\infty} \frac{f^{m}}{r_{3}^{m}}= & \frac{1}{\left(r_{3}-r_{1}\right)\left(r_{3}-r_{2}\right)}\left\{f^{2}(x)-\left(r_{1}+r_{2}\right) f(x)+r_{1} r_{2} x\right\} \\
& +\lim _{m \rightarrow+\infty} \frac{\left(\frac{r_{2}}{r_{3}}\right)^{m}}{\left(r_{2}-r_{1}\right)\left(r_{2}-r_{3}\right)}\left\{f^{2}(x)-\left(r_{1}+r_{3}\right) f(x)+r_{1} r_{3} x\right\} .
\end{aligned}
$$

Using the same idea and arguments as that of the case $1<r_{2}<-r_{1}<r_{3}$ in Theorem 4.1 , we get

$$
f^{2}(x)-\left(r_{1}+r_{2}\right) f(x)+r_{1} r_{2} x=0 .
$$

Hence every $C^{0}$ strictly decreasing solution $f$ of $(1.4)$ is a function in the class given in Theorem 3 of [14].

In the sequel we consider the case $1<r_{3}<-r_{1}<-r_{2}$. Let $f: \mathbb{R} \rightarrow \mathbb{R}$ be a $C^{0}$ strictly increasing solution of (1.4), the proof is the same as the case $1<-r_{1}<r_{3}<-r_{2}$. If $f: \mathbb{R} \rightarrow \mathbb{R}$ is a $C^{0}$ strictly decreasing solution of (1.4), then $f^{-1}$ is a solution of the dual equation (1.5). By Lemma 2.6 and the monotonicity of $f^{-1}$ we get

$$
f^{2}(x)-\left(r_{1}+r_{2}\right) f(x)+r_{1} r_{2} x=0
$$

by removing $1 / r_{3}$. Thus all $C^{0}$ strictly decreasing solutions of (1.4) are in the class given in Theorem 3 of [14].

The proof for the case $0<r_{3}<1<-r_{1}<-r_{2}$ is the same as that of the case $1<r_{3}<-r_{1}<$ $-r_{2}$. This completes the proof.

By considering the dual equation (1.5) we have the following corollary.

Corollary 4.3 Suppose that $0<-r_{1}<-r_{2}<r_{3}<1\left(0<-r_{1}<r_{3}<-r_{2}<1,0<r_{3}<-r_{1}<\right.$ $\left.-r_{2}<1,0<-r_{1}<-r_{2}<1<r_{3}\right)$. Iff $: \mathbb{R} \rightarrow \mathbb{R}$ is a $C^{0}$ solution of $(1.4)$, then $f^{-1}$ is a function in the class given in Theorem 4.3.

Theorem 4.4 Suppose that $0<-r_{1}<1<-r_{2}<r_{3}\left(0<-r_{1}<1<r_{3}<-r_{2}\right)$.

(i) Iff $: \mathbb{R} \rightarrow \mathbb{R}$ is a $C^{0}$ strictly increasing solution of $(1.4)$, then $f(x)=r_{3} x$.

(ii) Iff $: \mathbb{R} \rightarrow \mathbb{R}$ is a $C^{0}$ strictly decreasing solution of $(1.4)$, then $f(x)=r_{1} x$ or $f(x)=r_{2} x$. 
The proof is similar to that of $1<-r_{1}<-r_{2}<r_{3}\left(1<-r_{1}<r_{3}<-r_{2}\right)$ of Theorem 4.3. By using the dual equation (1.5) we have the following corollary.

Corollary 4.4 Suppose that $0<r_{3}<-r_{1}<1<-r_{2}\left(0<-r_{1}<r_{3}<1<-r_{2}\right)$. Iff $: \mathbb{R} \rightarrow \mathbb{R}$ is a $C^{0}$ solution of (1.4), then $f^{-1}$ is a function in the class given in Theorem 4.4.

\section{Further discussion and remarks}

As shown in the previous sections, we obtain all $C^{0}$ solutions of (1.4) for the cases listed in Tables 1-3. Consequently, all $C^{0}$ solutions of the 3rd-order equation (1.2) with $c \neq 0$ can be obtained by using Lemma 2.3. In what follows we prove some cases, listed in Tables 2 and 3 , which can also be generalized to the $n$ th-order polynomial-like iterative equation recursively. For example we consider the 4th-order equation (1.3) and take the first case $1<$ $-r_{1}<r_{2}<r_{3}$ in Table 2. Adding $r_{4}>1$ as the 4th characteristic root, we have the following result.

Theorem 5.1 Suppose that $1<-r_{1}<r_{2}<r_{3}<r_{4}\left(1<-r_{1}<r_{2}<r_{4}<r_{3}, 1<-r_{1}<r_{4}<r_{2}<\right.$ $\left.r_{3}\right)$.

(i) If $f: \mathbb{R} \rightarrow \mathbb{R}$ is a $C^{0}$ strictly increasing solution of the 4 th-order equation (1.3). Then $f$ is a solution given in Theorem 2 of [19] for the characteristic roots $r_{2}, r_{3}, r_{4}$.

(ii) If $: \mathbb{R} \rightarrow \mathbb{R}$ is a $C^{0}$ strictly decreasing solution of the 4 th-order equation (1.3). Then $f(x)=r_{1} x$.

Proof We first consider the case $1<-r_{1}<r_{2}<r_{3}<r_{4}$. Let $f: \mathbb{R} \rightarrow \mathbb{R}$ be a $C^{0}$ strictly increasing solution of the 4 th-order equation (1.3), then $f^{-1}$ is a $C^{0}$ strictly increasing solution of its dual equation. Making use of Lemma 2.5 and the monotonicity of $f^{-1}$ to remove $1 / r_{1}$, we get

$$
f^{-3}(x)-\left(\frac{1}{r_{2}}+\frac{1}{r_{3}}+\frac{1}{r_{4}}\right) f^{-2}(x)+\left(\frac{1}{r_{2} r_{3}}+\frac{1}{r_{2} r_{4}}+\frac{1}{r_{3} r_{4}}\right) f^{-1}(x)-\frac{1}{r_{2} r_{3} r_{4}} x=0,
$$

that is

$$
f^{3}(x)-\left(r_{2}+r_{3}+r_{4}\right) f^{2}(x)+\left(r_{2} r_{3}+r_{2} r_{4}+r_{3} r_{4}\right) f(x)-r_{2} r_{3} r_{4} x=0
$$

thus $f$ is a solution given in Theorem 2 of [19] for characteristic roots $r_{i}(i=2,3,4)$. On the other hand, assume that $f: \mathbb{R} \rightarrow \mathbb{R}$ is a $C^{0}$ strictly decreasing solution of the 4 th-order equation (1.3). By using Lemma 2.5 and the monotonicity of $f$ to remove $r_{4}$, we have

$$
f^{3}(x)-\left(r_{1}+r_{2}+r_{3}\right) f^{2}(x)+\left(r_{1} r_{2}+r_{1} r_{3}+r_{2} r_{3}\right) f(x)-r_{1} r_{2} r_{3} x=0 .
$$

Therefore, $f(x)=r_{1} x$ from Theorem 4.1.

The proof for the cases $1<-r_{1}<r_{2}<r_{4}<r_{3}$ and $1<-r_{1}<r_{4}<r_{2}<r_{3}$ is similar to the previous one and is omitted. This completes the proof.

Assuming that the characteristic root $r_{4}<-1$, we have the following result.

Theorem 5.2 Suppose that $1<-r_{1}<r_{2}<-r_{4}<r_{3}\left(1<-r_{1}<-r_{4}<r_{2}<r_{3}, 1<-r_{4}<-r_{1}<\right.$ $\left.r_{2}<r_{3}\right)$. 
(i) If $f: \mathbb{R} \rightarrow \mathbb{R}$ is a $C^{0}$ strictly increasing solution of the 4 th-order equation (1.3). Then $f$ is a solution given in Theorem 4.1.

(ii) Iff $: \mathbb{R} \rightarrow \mathbb{R}$ is a $C^{0}$ strictly decreasing solution of the 4 th-order equation (1.3). Then $f$ is a solution given in Theorem 4.3 .

The proof is similar to that of Theorem 5.1 and is omitted.

Using the same idea and arguments as previous, we can obtain all $C^{0}$ solutions of the 4thorder equation (1.3) for some cases listed in Tables 2 and 3 by adding the 4th characteristic root $r_{4}$. In general, for those cases that the characteristic root with the largest module has different sign from the ones with the smallest module, we can obtain all $C^{0}$ solutions of the $n$ th-order polynomial-like iterative equation by using that of the $n-1$ th-order polynomiallike iterative equation without essential difficulty.

\section{Competing interests}

The authors declare that they have no competing interests.

\section{Authors' contributions}

The authors completed the paper together. They also read and approved the final manuscript.

\section{Acknowledgements}

The authors sincerely appreciate the referee for his/her excellent comments and suggestions, which have improved the presentations of the paper. Project was supported by Scientific Research Fund of Shandong Provincial Education Departments (J12L59), Scientific Research Fund of Sichuan Provincial Education Departments (12ZA086) and Doctoral Fund of Binzhou University (2013Y04)

Received: 29 September 2014 Accepted: 30 November 2014 Published: 15 Dec 2014

\section{References}

1. Braon, K, Jarczyk, W: Recent results on functional equations in a single variable, perspectives and open problems. Aequ. Math. 61, 1-48 (2001)

2. Targoński, G: Topics in Iteration Theory. Vandenhoeck \& Ruprecht, Göttingen (1981)

3. Kuczma, M, Choczewski, B, Ger, R: Iterative Functional Equations. Encyclopedia Math. Appl., vol. 32. Cambridge University Press, Cambridge (1990)

4. Ng, CT, Zhang, W: Invariant curves for planar mappings. J. Differ. Equ. Appl. 3, 147-168 (1997)

5. Chen, J, Zhang, W: Leading coefficient problem for polynomial-like iterative equations. J. Math. Anal. Appl. 349 , 413-419 (2009)

6. Kulczycki, M, Tabor, J: Iterative functional equations in the class of Lipschitz functions. Aequ. Math. 64, 24-33 (2002)

7. Tabor, J, Zoldak, M: Iterative equations in Banach spaces. J. Math. Anal. Appl. 229, 651-662 (2004)

8. Trif, T: Convex solutions to polynomial-like iterative equations on open intervals. Aequ. Math. 79, 315-325 (2010)

9. Xu, B, Zhang, W: Construction of continuous solutions and stability for the polynomial-like iterative equation. J. Math. Anal. Appl. 325, 1160-1170 (2007)

10. Zhang, $W$ : On the differentiable solutions of the iterated equation $\sum_{i=1}^{n} \lambda_{i} f^{i}(x)=F(x)$. Nonlinear Anal. 15, 387-398 (1990)

11. Zhang, W: Solutions of equivariance for a polynomial-like iterative equation. Proc. R. Soc. Edinb. A 130, 1153-1163 (2000)

12. Murugan, V, Subrahmanyam, PV: Existence of continuous solutions for an iterative functional series equation with variable coefficients. Aequ. Math. 78, 167-176 (2009)

13. Murugan, $V$, Subrahmanyam, PV: Existence of solutions for equations involving iterated functional series. Fixed Point Theory Appl. 2005(2), 219-232 (2005)

14. Nabeya, S: On the functional equation $f(p+q x+r f(x))=a+b x+c f(x)$. Aequ. Math. 11, 199-211 (1974)

15. Matkowski, J: The twenty-sixth international symposium on functional equations, Remark 35. Report of meeting. Aequ. Math. 37, 119 (1989)

16. Jarczyk, W: On an equation of linear iteration. Aequ. Math. 51, 303-310 (1996)

17. Tabor, J, Tabor, J: On a linear iterative equation. Results Math. 27, 412-421 (1995)

18. Matkowski, J, Zhang, W: Method of characteristic for functional equations in polynomial form. Acta Math. Sin. 13(3), 421-432 (1997)

19. Yang, D, Zhang, W: Characteristic solutions of polynomial-like iterative equations. Aequ. Math. 67, 80-105 (2004)

20. Zhang, W, Zhang, W: On continuous solutions of $n$-th order polynomial-like iterative equations. Publ. Math. (Debr.) 76(1-2), 117-134 (2010)

21. Morawiec, J: Around a problem of Nicole Brillouët-Belluot. Aequ. Math. (2014). doi:10.1007/s00010-013-0216-8

22. Morawiec, J: Around a problem of Nicole Brillouët-Belluot, II. Aequ. Math. (2014). doi:10.1007/s00010-013-0249-z

23. Matkowski, J, Zhang, W: Characteristic analysis for a polynomial-like iterative equation. Chin. Sci. Bull. 43(3), $192-196$ (1998) 
10.1186/1687-1847-2014-318

Cite this article as: Zhang and Gong: Continuous solutions of 3rd-order iterative equation of linear dependence. Advances in Difference Equations 2014, 2014:318

Submit your manuscript to a SpringerOpen ${ }^{\circ}$ journal and benefit from:

- Convenient online submission

- Rigorous peer review

- Immediate publication on acceptance

- Open access: articles freely available online

- High visibility within the field

- Retaining the copyright to your article

Submit your next manuscript at $\gg$ springeropen.com 